$4 \mathrm{~cm} ; 2$ (25\%) had a 1a1 stage. No patient had a tumor larger than $4 \mathrm{~cm}$ on examination, MRI, and histological examination. No patient received neoadjuvant chemotherapy. 2 patients (25\%) underwent a biopsy for the purpose of establishing a diagnosis, 6 patients (75\%) conization of the cervix. The average age of the patients was 28 years (from 26 to 37 years). $5(62.5 \%)$ patients were nulliparous, 2 patients had 2 children, 1 patient had 1 child.

LVSI was negative in preoperative histological examination, and resection margins were also negative. In 1 patient $(12.5 \%)$ after histological examination LVSI was positive, in 7 it was negative. Of the 8 patients who retained fertility, there were 5 pregnancies, 2 miscarriages at 9-10 weeks, and 3 deliveries at 36-37 weeks of gestation.

Conclusion* Thus, in 2013-2021, 8 radical trachelectomy operations were successfully performed. The data presented in this publication demonstrate that patients with stage IB1 tumors ranging in size from 2 to $4 \mathrm{~cm}$ and with favorable histology are acceptable candidates for attempted radical trachelectomy. These patients must be clearly informed of all the options if they want to consider maintaining fertility.

\section{SINGLE INSTITUTION OUTCOMES FOR CERVICAL CANCER PATIENTS TREATED WITH PALLIATIVE SYSTEMIC ANTI CANCER THERAPY}

${ }^{1} \mathrm{C}$ Cotronei ${ }^{*},{ }^{2} \mathrm{~A}$ Stillie, ${ }^{2} \mathrm{M}$ Zahra. ${ }^{1}$ University of Edinburgh, UK; ${ }^{2}$ Western General Hospital Edinburgh Cancer Centre, Edinburgh, UK

10.1136/ijgc-2021-ESGO.4

Aim Bevacizumab, in combination with chemotherapy, has been implemented as first line treatment of patients with metastatic cervical cancer since 2015. This is a single institution review to evaluate the overall survival (OS) and progression free survival (PFS) for patients with advanced cervical cancer treated with palliative systemic anti cancer therapy (SACT) over a 5-year period from 2015-2020.

Methodology Patient records were reviewed retrospectively for all cervical cancer patients who received palliative SACT in a single institution between 2015-2020. Patient demographics, previous treatment for cervical cancer (if applicable), palliative SACT regimen received, toxicities, and outcome data were recorded. OS and PFS were calculated for the whole cohort and for the following subgroups: patients treated with Bevacizumab in combination with palliative chemotherapy; patients treated with chemotherapy alone. Toxicities were recorded by Common Toxicity Criteria (CTC).

Result(s)* 52 patients received palliative SACT during the 5year period following a diagnosis of metastatic cervical cancer. The median age was 51 (range 25-76). The majority of patients had a squamous cell histology (75\%). 40 patients received Bevacizumab in combination with chemotherapy, 12 were treated with chemotherapy alone due to comorbidities. The median OS for the whole patient cohort was 64 weeks and PFS was 45 weeks. Patients who received bevacizumab in combination with chemotherapy had a median OS of 76 weeks compared to 50 weeks in the chemotherapy only subgroup. Grade 1 and 2 toxicites were comparable in patients receiving chemotherapy alone and those treated with Bevacizumab. $10 \%$ of patients in the cohort receiving bevacizumab developed a thromboembolic event compared with 5.5\% in the chemotherapy only cohort.
Conclusion* Results from this study of real world data are comparable with the published data. The results support the continued use of Bevacizumab, in combination with chemotherapy, in our patient population as the combination regimen improves OS with acceptable toxicties.

\section{COMPARISON OF LOCALLY ADVANCED CERVICAL CANCER TREATMENT GUIDELINES IN EUROPE}

${ }^{1} \mathrm{~A}$ Leary*, ${ }^{2} \mathrm{~B}$ Monk, ${ }^{3} \mathrm{~J}$ Takyar, ${ }^{4} \mathrm{~A}$ Nunes, ${ }^{4} \mathrm{JD}$ Hernández Chagüi, ${ }^{4} \mathrm{~K}$ Rabon-Stith, ${ }^{5}$ E Pujade-Lauraine. 'Gustave Roussy Cancer Center, University Paris Saclay, Department of Medical Oncology, Villejuif, France; ' ${ }^{2}$ University of Arizona College of Medicine, Creighton University School of Medicine, Division of Gynecologic Oncology, Phoenix, USA; ${ }^{3}$ Parexel International, HEOR Evidence Evaluation Regulatory and Access, Mohali, India; ${ }^{4}$ AstraZeneca, Gaithersburg, USA; ${ }^{5}$ Arcagy-Gineco, Paris, USA

\subsection{6/ijgc-2021-ESGO.5}

Introduction/Background* From our previously conducted global systematic literature review (SLR) assessing recommended treatment for locally advanced cervical cancer (LACC) it was observed that there was international consensus on the use of concurrent chemoradiotherapy (cCRT) as standard of care (SoC) for Stage IIB-IVA LACC, but recommendations for Stage IB2-IIA LACC varied. Here, we present a subanalysis of European guidelines to determine if recommended LACC treatment is consistent across Europe.

Methodology English-language cervical cancer treatment guidelines and consensus statements were identified through literature databases (1999-2020), international and national health organizations, and general internet searches. Included guidelines represented the latest updates from Europe. Non-English guidelines from EU-5 countries were translated for comparison (Italian [AIOM], German [S3], and French [HAS]) to the 4 European guidelines obtained through this SLR protocol (ESTRO/ESGO/ESP, ESMO, Spain [SEOM], UK [NHS]). An English-language guideline from Germany was excluded because a newer German-language only version was available. Result(s)* The 7 guidelines were updated between 2010 and 2021 (table 1). ESTRO/ESGO/ESP guidelines used AJCC 8th edition TNM criteria for staging. All others, except SEOM, used FIGO 2009 criteria; SEOM used FIGO 2018 criteria. Table 1 shows recommended treatment by LACC stage. For Stage IB2-IIA2 disease, cCRT was the SoC or an alternative to radical hysterectomy. AIOM was the only guideline to recommend radiotherapy alone for Stage IIA1 disease. cCRT followed by brachytherapy was SoC for suitable patients with Stage IIB-IVA LACC among all European guidelines. For cCRT, a platinum-based regimen was recommended by all, and most guidelines recommended an external beam radiation therapy (EBRT) dose of 45-50 Gy (table 2).

Conclusion* In line with global SLR findings, consensus on the use of cCRT as primary treatment for Stage IIB-IVA LACC was recommended by European guidelines. The ideal cCRT regimen was weekly cisplatin at $40 \mathrm{mg} / \mathrm{m} 2$ concurrent with EBRT at 45-50 Gy and followed by brachytherapy. For Stage IB-IIA LACC, recommendations mainly varied between radical hysterectomy and cCRT as options or cCRT alone. Alignment of guidelines with FIGO 2018 staging criteria may help reduce variation in recommended treatment for earlystage LACC.

Funding AstraZeneca

Klikně te nebo klepně te sem a zadejte text. 
Abstract 106 Table 1 Recommended standard of care by LACC stage in European guidelines

\begin{tabular}{|c|c|c|c|c|c|}
\hline \multirow{2}{*}{$\begin{array}{l}\text { Guideline / } \\
\text { Year of last } \\
\text { update }\end{array}$} & \multicolumn{5}{|c|}{ LACC Stage } \\
\hline & IB2 & IB3 & $\| \mathrm{A} 1$ & IIA2 & $\begin{array}{l}\text { IIB- } \\
\text { IVA }\end{array}$ \\
\hline \multirow{2}{*}{$\begin{array}{l}\text { ESMO } \\
2017\end{array}$} & $\begin{array}{c}\checkmark \\
\text { (non-bulky) }\end{array}$ & & $\begin{array}{c}\checkmark \\
\text { (non-bulky) }\end{array}$ & $\begin{array}{c}\checkmark \\
\text { (non-bulky) }\end{array}$ & \multirow[b]{2}{*}{$\checkmark$} \\
\hline & $\begin{array}{c}\checkmark \\
\text { (bulky, or non-bulky } \\
\text { and inoperable) }\end{array}$ & & $\begin{array}{c}\checkmark \\
\text { (bulky, or non-bulky } \\
\text { and inoperable) }\end{array}$ & $\begin{array}{c}\checkmark \\
\text { (bulky, or non-bulky } \\
\text { and inoperable) }\end{array}$ & \\
\hline $\begin{array}{c}\text { ESTRO/ } \\
\text { ESGO/ESP } 2018\end{array}$ & (T1b2 LN+ only) & & $\begin{array}{l}\checkmark \\
\text { (T2a1, LN+ only, } \\
\text { BT not indicated) }\end{array}$ & $\begin{array}{c}\checkmark \\
\text { (T2a2, LN+ only) }\end{array}$ & $\checkmark$ \\
\hline \multirow{2}{*}{$\begin{array}{l}\text { NHS } \\
\text { (UK) } \\
2018\end{array}$} & \multirow{2}{*}{$\checkmark$} & & $\begin{array}{c}\checkmark \\
\text { (non-bulky) }\end{array}$ & $\begin{array}{c}\checkmark \\
\text { (non-bulky) }\end{array}$ & \multirow{2}{*}{$\checkmark$} \\
\hline & & & $\begin{array}{c}\checkmark \\
\text { (bulky or non-bulky) }\end{array}$ & $\begin{array}{c}\checkmark \\
\text { (bulky or non-bulky) }\end{array}$ & \\
\hline $\begin{array}{c}\text { HAS (France) } \\
2010\end{array}$ & $\checkmark$ & & $\checkmark$ & $\checkmark$ & $\checkmark$ \\
\hline \multirow{2}{*}{$\begin{array}{c}\text { S3 (Germany) } \\
2021\end{array}$} & $\checkmark$ & & $\checkmark$ & $\checkmark$ & \multirow{2}{*}{$\checkmark$} \\
\hline & $\begin{array}{c}\checkmark \\
\text { (LN+ or inoperable) }\end{array}$ & & $\begin{array}{c}\checkmark \\
\text { (LN+ or inoperable) }\end{array}$ & $\begin{array}{c}\checkmark \\
\text { (LN+ or inoperable) }\end{array}$ & \\
\hline \multirow{2}{*}{$\begin{array}{l}\text { AIOM (Italy) } \\
2019\end{array}$} & $\checkmark$ & & $\checkmark$ & \multirow[t]{2}{*}{$\checkmark$} & \multirow[t]{2}{*}{$\checkmark$} \\
\hline & $\checkmark$ & & $\checkmark$ & & \\
\hline \multirow{2}{*}{$\begin{array}{l}\text { SEOM } \\
\text { (Spain) } \\
2019\end{array}$} & $\checkmark$ & \multirow{2}{*}{$\checkmark$} & $\checkmark$ & \multirow[b]{2}{*}{$\checkmark$} & \multirow{2}{*}{$\checkmark$} \\
\hline & $\begin{array}{c}\checkmark \\
\text { (inoperable) }\end{array}$ & & $\begin{array}{c}\checkmark \\
\text { (inoperable) }\end{array}$ & & \\
\hline
\end{tabular}

\section{Radical hysterectomy $\quad$ CCRT+ brachytherapy Radiotherapy alone}

$\checkmark$ Recommended treatment option; AIOM, Associazione Italiana di Oncologica Medica; BT, brachytherapy; ESMO, European Society for Medical Oncology; ESTRO, European Society for Radiotherapy and Oncology; ESGO, European Society for Gynaecological Oncology; ESP, European Society of Pathology; HAS, Haute Autorité de Santé; LACC, locally advanced cervical cancer; LN, lymph node; S3, refers to consensus-based medical guidelines developed in collaboration with the German Cancer Society (DKG) German Cancer Aid (DKH) and Working Group of the Scientific Medical Societies (AWMF) in Germany; SEOM, Spanish Society of Medical Oncology.

Abstract 106 Table 2 Details of cCRT regimens

\begin{tabular}{|c|c|c|c|c|c|c|c|}
\hline $\begin{array}{l}\text { cCRT regimen } \\
\text { components }\end{array}$ & ESMO & $\begin{array}{c}\text { ESTRO/ESGOI } \\
\text { ESP }\end{array}$ & SEOM & NHS & HAS & S3 & AIOM \\
\hline Chemotherapy & $\begin{array}{l}\text { Weekly } \\
\text { Cisplatin } \\
40 \mathrm{mg} / \mathrm{m}^{2}\end{array}$ & $\begin{array}{l}\text { Weekly } \\
\text { Cisplatin } \\
40 \mathrm{mg} / \mathrm{m}^{2}\end{array}$ & $\begin{array}{l}\text { Weekly } \\
\text { Cisplatin } \\
40 \mathrm{mg} / \mathrm{m}^{2}\end{array}$ & Cisplatin & $\begin{array}{l}\text { Platinum-based } \\
\text { therapy, 5-6 } \\
\text { courses }\end{array}$ & $\begin{array}{l}\text { Weekly } \\
\text { Cisplatin } \\
40 \mathrm{mg} / \mathrm{m}^{2}\end{array}$ & $\begin{array}{l}\text { Weekly } \\
\text { Cisplatin } \\
40 \mathrm{mg} / \mathrm{m}^{2}\end{array}$ \\
\hline $\begin{array}{c}\text { EBRT dose } \\
\text { 40-50 Gy }\end{array}$ & $\begin{array}{c}\text { Not } \\
\text { discussed }\end{array}$ & $\checkmark$ & $\checkmark$ & $\checkmark$ & Not discussed & $\checkmark$ & $\checkmark$ \\
\hline $\begin{array}{c}\text { Brachytherapy } \\
\text { dose }\end{array}$ & $\begin{array}{c}\text { Not } \\
\text { discussed }\end{array}$ & $\begin{array}{l}40-45 \mathrm{~Gy} \\
\text { (EQD2) }\end{array}$ & $\begin{array}{c}5.5-8 \text { Gy by } \\
3 \text { to } 5 \\
\text { fractions/ } \\
\text { week }\end{array}$ & $\begin{array}{l}7 \text { Gy } \times 3 \text { to } \\
\text { point } A \\
\text { over } 10 \\
\text { days }\end{array}$ & Not discussed & $\begin{array}{l}\text { 40- } 50 \text { Gy } \\
\text { (EQD2) }\end{array}$ & $\begin{array}{c}\text { 25-30 Gy HDR } \\
\text { (equivalent to } \\
40 \text { Gy LDR) }\end{array}$ \\
\hline Total RT dose & $\begin{array}{c}\text { Not } \\
\text { discussed }\end{array}$ & $\begin{array}{l}\geq 85-90 \text { Gy } \\
\text { EQD2 to the } \\
\text { adaptive high- } \\
\text { risk clinical } \\
\text { target volume }\end{array}$ & $\begin{array}{c}\text { Not } \\
\text { discussed }\end{array}$ & $\begin{array}{c}\text { Not } \\
\text { discussed }\end{array}$ & $\begin{array}{c}\text { Not } \\
\text { discussed }\end{array}$ & $\geq 85$ Gy & $\begin{array}{c}80 \text { Gy for IB1- } \\
\text { IIA1, 90-95 Gy } \\
\text { for larger } \\
\text { tumours }\end{array}$ \\
\hline
\end{tabular}

